\title{
Study of the Designed Washing Machine for Canning Industry
}

\author{
Andrey V. Mayorov ${ }^{1, *}$ \\ ${ }^{1}$ Associate Professor, Mari State University, Yoshkar-Ola, Russia
}

\begin{abstract}
Nowadays industry uses special washing machines to wash the outer surface of cans, which can be jet, submersible and combined-type. These washing machines are quite energy-intensive and metalconsuming. A washing machine was designed in Mari State University, in which the objects cleaned gain eccentric motion and bubbling is created from the side of cylindrical cans. In order to identify the dependence of the cleaning quality on liquid activation using the air bubbling method, a number of factorat-a-time experiments with a three-fold repeatability were performed at the outside of the washed objects. Special theoretical calculations were carried out to identify the limits of the drive wheel speed values aimed at studying kinematic parameters. The type of hyperbolic dependence of the wheel diameter on its rotation frequency is also determined.
\end{abstract}

\section{Introduction}

Quite a long time has passed since the canned foods became the most demanded food products, and the level of demand for them increases from year to year. The range of canned products is also constantly expanding. High demand level is caused by asepticity, convenient storage and transportation, the possibility of quick use in the cooking process. A high demand level for canned foods requires the implementation of measures for the further improvement of the production process of this food product category. The sales volume in the face of stiff competition between manufacturers directly depends on the appearance of a can and the completeness of information on the label. The label not only serves to inform customers, but also acts as an advertisement, for which reason it shall meet the conditions of cleanliness, integrity, density, firmly covering the entire can body. High-quality labeling requires the cans to be clean, dry and free of fat. In view of the above conditions, a need emerges to include the washing of filled cans in the canning production line [15].

Nowadays, special washing machines are used in the process of washing the outer surface of cans, which can be jet, submersible and combined. These washing machines are quite energy-intensive and metalconsuming, which implies high operating costs for the food industry. For this reason, the design and launch of innovative and highly efficient washing machines in the canning industry is a sufficient reserve that allows to sufficiently reduce energy and material costs, as well as the cost of the canning process [6-9].

A washing machine was designed in Mari State University, in which the cleaned objects gain eccentric motion and bubbling is created from the side of cylindrical cans.

\section{Materials and Methods}

In order to determine the relationship between the indicators of quality of cleaning cans from liquid activation by air bubbling from the outside of the objects being washed, a series of factor-at-a-time experiments with three-fold repeatability were performed using a 2 $\mathrm{g} / \mathrm{l}$ detergent concentration (as recommended by the developer of the Nika-2 detergent). The type of detergent in mechanized washing method was selected in accordance with the recommendations of SanPiN 2.3.4.050-96 and the instructions on the cleaning of equipment, inventory and packaging units within the food industry enterprises. Sanitary and epidemiological rules and norms (SanPiN) are state by-laws and regulations with descriptions and requirements of safe and harmless to human, human populations and descendants of environmental factors and their optimal and safe quantitative parameters in order to maintain health and normal life. With the purpose of identifying the limits of the drive wheel speed values, a number of theoretical calculations were made to analyze the kinematic parameters, as well as the recommendations of other research scientists were used [10-16]. According to them, the optimal movement speed of the objects being cleaned in the solution varies from 0.3 to $0.85 \mathrm{~m} / \mathrm{s}$.

The can speed $(\mathrm{m} / \mathrm{s})$ in the designed washing machine in the first and second sections was calculated by substituting the initial data into the following expression (1):

$$
V_{b}=\frac{f_{1} \cdot Q \cdot R^{2} \cdot \omega_{k} \cdot\left(1-\eta_{b}\right)}{\frac{\varepsilon \cdot d}{2 \cdot \sqrt[3]{k}} \cdot \sqrt[3]{\frac{\left(Q+G_{b}\right)^{4}}{d^{2} \cdot b}}}
$$

\footnotetext{
* Corresponding author: ao maiorov@mail.ru
} 
where $f_{1}$ is the friction factor of the wheel rim rubber with a can;

$Q$ is the wheel pressure on the can, $\mathrm{N}$;

$R$ is the wheel radius, $\mathrm{m}$;

$\omega_{k}$ is the drive wheel angular velocity, $\mathrm{rad} / \mathrm{s}$;

$\eta_{b}$ is the coefficient of slipping of the drive wheel;

$\varepsilon$ is the correction factor;

$d$ is the can diameter, m;

$k$ is the coefficient of volumetric shearing of the guide material, $\mathrm{N} / \mathrm{m}^{3}$;

$Q$ is the wheel pressure on the can, $\mathrm{N}$;

$G_{b}$ is the weight of the filled can, N;

$b$ is the can width, m;

$d$ is the can diameter, $\mathrm{m}$

The washing machine wheel diameter was determined based on the following expression (2)

$$
D=2 R=\frac{d}{30} \sqrt{\frac{\frac{7 W \cdot \varepsilon}{12 \sqrt[3]{k}} \cdot \sqrt[3]{\frac{\left(Q+G_{b}\right)^{4}}{d^{2} \cdot b}}}{\tau \cdot \sigma \cdot f_{1} \cdot Q \cdot \omega_{k} \cdot\left(1-\eta_{b}\right)}}
$$

where $\mathrm{W}$ is the machine performance, $\mathrm{pcs} / \mathrm{h}$;

$\tau$ is the use coefficient;

$\sigma$ is the coefficient that takes into account the number of sections in the machine;

Accordingly, the most rational wheel diameter is dependent on the washing machine performance, the drive wheel speed, the pressure of the wheel rim on the can, the weight and dimensions of cans, the coefficient of friction between the wheel rim and the can, and the physical and mechanical properties of the guides and detergent.

In accordance with expression (2), diagrams were plotted of the dependence of the wheel diameter on the speed at different values of the washing machine performance (Fig. 1).

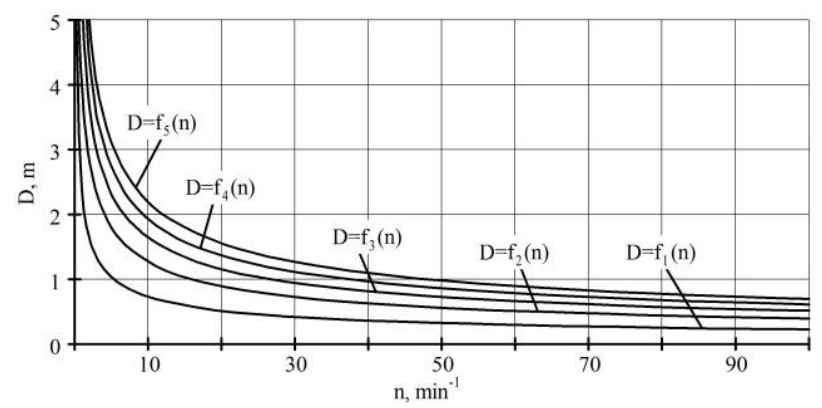

Fig. 1. Dependence of the wheel diameter on its speed. at various values of the washing machine performance: $\left(D=f_{1}(n)\right.$ - performance of $2.000 \mathrm{pcs} / \mathrm{h} ; \mathrm{D}=\mathrm{f}_{2}(\mathrm{n})$ - performance of $6.000 \mathrm{pcs} / \mathrm{h} ; \mathrm{D}=\mathrm{f}_{3}(\mathrm{n})$ - performance of $10.000 \mathrm{pcs} / \mathrm{h} ; \mathrm{D}=\mathrm{f}_{4}(\mathrm{n})$ - performance of $14.000 \mathrm{pcs} / \mathrm{h} ; \mathrm{D}=\mathrm{f}_{5}(\mathrm{n})$ - performance of $18.000 \mathrm{pcs} / \mathrm{h})$.

After analyzing the data in Fig. 1, we can conclude that the dependence of the wheel diameter on its speed is hyperbolic. The higher the washing machine performance, the higher will be the wheel diameter at the same speed. The higher the performance of the machine, the more important is its speed compared to the diameter of its wheel.

Within the rotation speed range of $0-60 \mathrm{~min}^{-1}$, in case of obtaining any required productivity value up to $18.000 \mathrm{pcs} / \mathrm{h}$, it is necessary to sharply increase the wheel diameter. If the rotation speed exceeds $60 \mathrm{rpm}$, reduction of the wheel diameter required is insignificant. The minimum wheel diameter of $0.5 \mathrm{~m}$ is obtained at a speed of $60 \mathrm{rpm}$ and at a minimum productivity of $6.000 \mathrm{pcs} / \mathrm{h}$. Any performance up to $18.000 \mathrm{pcs} / \mathrm{h}$ can be obtained with the wheel diameter of than $0.71 \mathrm{~m}$ by altering the speed.

The larger the wheel diameter, the longer time it will take the objects to be cleaned in the detergent and the larger will be the overall dimensions of the machine, and the increase in speed increases the drive power and, consequently, the wheel diameter shall vary between 0.5 and $0.8 \mathrm{~m}$. The designed washing machine has the wheel diameter of $0.71 \mathrm{~m}$.

Fig. 2 shows diagrams of the values of the can speed depending on the speed of the drive and idle wheels. At the same time, the recommended values [15-16] of the speed of the objects being cleaned in the detergent are given. Fig. 2 suggests that the limits of the wheel speed that will correspond to the recommendations range between $\mathrm{n}_{\min } \approx 15 \mathrm{~min}^{-1}$ and $\mathrm{n}_{\max } \approx 35 \mathrm{~min}^{-1}$.

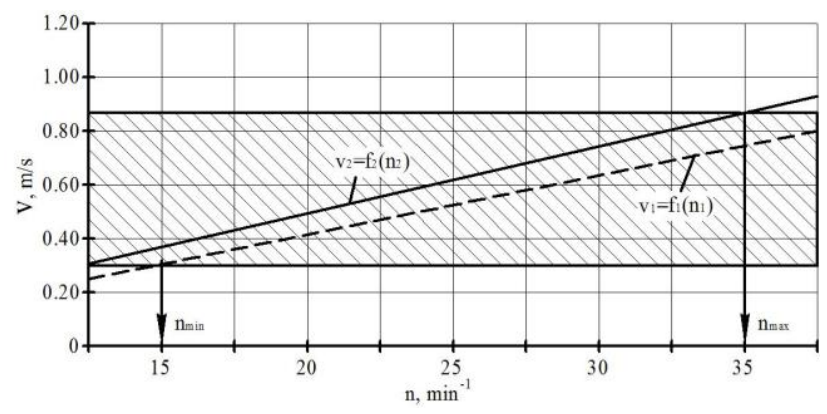

Fig. 2. Diagrams of the can speed dependence on the speed of the drive (- - $)$ and idle (-

The following equipment was used for measuring the parameters of the analyzed factors: the drive wheel speed was set using the ATV31HU15N4 frequency converter, the detergent volume was calculated using the VLKT $500 \mathrm{~g}$ analytic balance, the detergent temperature in the tank was determined using the Metacon 512-P-TS100-1 measurement controller.

\section{Results and Discussion}

Results of the factor-at-a-time experiments are shown in Fig. 3. After analyzing these results, we can conclude that the cleaning quality indicators increase with temperature and the drive wheel speed. Cleaning quality also improves when using the liquid activation method that involves air bubbling from the outside of the objects being washed.

Washing indicators increase with the drive wheel speed up to $n=35 \mathrm{~min}^{-1}$. This occurs due to the fact that the higher the wheels speed, the higher the surface hydrodynamic forces (drag and tangential drag forces of 
a liquid), which are designed to clean the surface of an object.



a

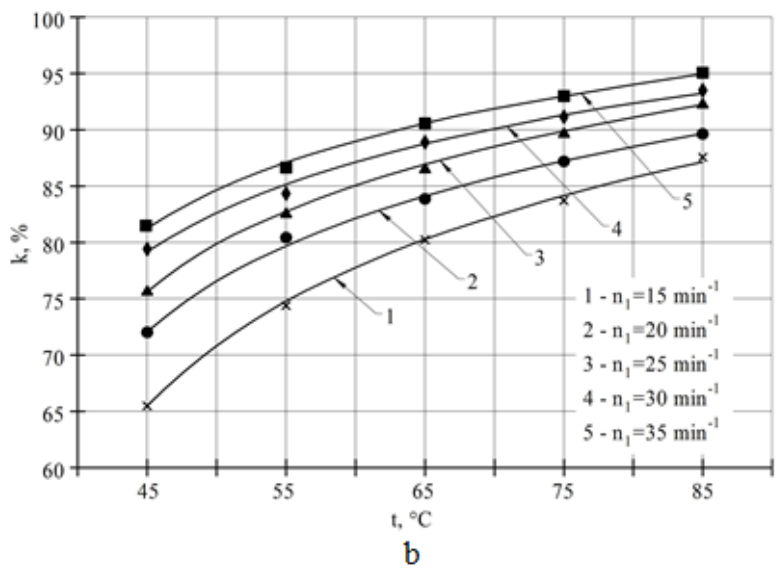

Fig. 3. Dependences of the can surface cleaning quality on the detergent temperature at various washing machine drive wheel speeds ( $\mathrm{a}$ - no bubbling; $\mathrm{b}$ - bubbling).

The temperature dependence of the can cleaning quality was analyzed at various rotation frequencies, based on which we can conclude that the cleaning quality indicators with bubbling rapidly increase at lower temperatures and minimum speeds. In particular, at $\mathrm{t}=$ $45{ }^{\circ} \mathrm{C}$ and $\mathrm{n}=15 \mathrm{~min}^{-1}$, the cleaning quality indicators reach $65 \%$ with bubbling, and as little as $14 \%$ without bubbling. Accordingly, it is possible to ensure highquality surface cleaning and save the detergent and the energy required for wheel rotation.

\section{Conclusions}

According to the observed results, as part of the washing machine operation, the use of the liquid activation method by air bubbling from the outside of the objects being washed causes cavitation, in which air bubbles enter the powerful turbulent liquid flows. These air bubbles mix with the liquid and burst upon hitting the objects being cleaned, thus applying additional pressure on the contaminated areas. For this reason, the cleaning quality indicators are much higher when using the bubbler.

\section{References}

[1] M. Mohayeji, et al., J. Taiwan Inst. Chem. E., 60, 76-82 (2016).

[2] S. Rauf, et al., Procedia Comput. Sci., 83, 902906 (2016).

[3] J.R. McDonough, et al., Chem. Eng. J., 265, 10121 (2015).

[4] M.A. Abdel-Fatah, et al., Ain Shams Engineering Journal, 8 (3), 305-310 (2017).

[5] Z. Chen, et al., Chem. Eng. Sci., 130, 254-263 (2015).

[6] O.V. Galtseva, et al., IOP Conf. Ser.: Mater. Sci. Eng., 132, 012003 (2016). DOI: 10.1088/1757899X/132/1/012003.

[7] J. Hodgkinson, R.P. Tatam, Meas. Sci. Technol., 24, 1 (2013).

[8] A.V. Yurchenko, et al., Journal of Physics: Conference Series, 88, 1, 012034 (2017).

[9] V.C. Eze, et al., Chem. Eng. J., 322, 205-214 (2017).

[10] M.G. Tadesse, et al., Smart Mater Struct, 26, 065016 (2017).

[11] H.S. Wahad, et al., IOP Conf. Ser.: Mater. Sci. Eng., 174, 012021 (2017). DOI: 10.1088/1757899X/174/1/012021.

[12] M. Mohayeji, et al., J. Taiwan. Inst. Chem. E., 60, 76-82 (2016).

[13] Z. Chen, et al., Chem. Eng. Sci., 130, 254-263 (2015).

[14] V.N. Borikov, et al., J. Phys. Conf. Ser., 671, 1, $012014 \quad$ (2016). DOI: 10.1088/17426596/671/1/012014.

[15] A.V. Mayorov, et al., IOP Conf. Ser.: Mater. Sci. Eng., 516, 012012 (2019). DOI: 10.1088/1757899X/516/1/012012.

[13] A.V. Mayorov, et al., IOP Conf. Ser.: Mater. Sci. Eng., 457, 012001 (2018). DOI: 10.1088/1757$899 X / 457 / 1 / 012001$. 